\title{
Null Steering of Circular Array Using Array Factor for GPS Anti-Jam
}

\author{
Taek-Sun Kwon ${ }^{1} \cdot$ Jae-Gon Lee ${ }^{2, *} \cdot$ Jeong-Hae Lee ${ }^{1, *}$
}

\begin{abstract}
In this letter, the null steering of a circular array is presented using a modified array factor (AF) for a global positioning system (GPS) anti-jam. The seven radiating elements were designed using a mu-zero resonance (MZR) circularly polarized (CP) antenna arranged toward the center. Since the radiating elements, which are arranged toward the center, have a $\mathrm{CP}$ characteristic, the $\mathrm{AF}$ of the seven radiating elements has to be modified considering the rotation angle of the nth radiating element. The phases of input ports can be calculated to implement a nulling of radiation patterns where the modified AF is zero. To verify the modified AF for null steering in the desired direction, two cases of power dividers operating in $L_{2}$ band $(1.2276 \mathrm{GHz})$ were fabricated to achieve pattern nulling at a certain angle. The modified AF can be confirmed by a comparing the simulated and measured radiation patterns.
\end{abstract}

Key Words: Anti-Jam, Array, Circular Array, Null Steering.

\section{INTRODUCTION}

As location tracking technology has developed, reliance on global positioning system (GPS) communication has increased. Currently, anti-jamming is researched as protection against environmental factors such as distortion, interferences caused by multipath propagation, and jamming signals that intentionally disrupt communication. The controlled reception pattern antenna (CRPA) array is used to control nulling in the direction of a jamming signal and increase gain in the direction of the satellites [1-3]. It is advantageous to design many radiating elements to protect GPS communications against jamming signals because the number of nulls $(N-1)$ is dependent on the number of radiating elements $(N)$. Thus, it is essential to design a miniaturized radiating element for multiple nulls.

There are various numerical methods to achieve null steering in a specific direction [3, 4]. However, null steering using nu- merical methods is both inaccurate and time-consuming if cost function is not elaborated. On the other hand, a total radiation pattern can be obtained from an active element pattern (AEP) multiplied by the array factor (AF). Using an AF, the input phases of radiating elements can be simply calculated to obtain nulls in the directions of jamming signals.

In this letter, the AF of a circular array should be modified because the radiating elements are arranged toward the center. In addition, the radiating elements are designed using mu-zero resonance (MZR), which is suitable for a miniaturization to implement the seven elements in a 5-inch circle space [5]. Since the radiating elements have a CP characteristic for GPS communication, the $\mathrm{AF}$ of the seven radiating elements can be modified considering the rotation angle of the $n^{\text {th }}$ radiating element. From the modified AF, the phases of input ports can be obtained to achieve pattern nulling at a certain angle. The one CRPA circular array and the two kinds of power dividers used

Manuscript received February 7, 2018 ; Accepted April 26, 2018 ; Accepted June 19, 2018. (ID No. 20180207-014J)

${ }^{1}$ School of Electronic and Electrical Engineering, Hongik University, Seoul, Korea.

${ }^{2}$ Metamaterial Electronic Device Research Center, Hongik University, Seoul, Korea.

"Corresponding Author: Jae-Gon Lee (e-mail: jaegonlee@hongik.ac.kr), Jeong-Hae Lee (e-mail: jeonglee@hongik.ac.kr)

This is an Open-Access article distributed under the terms of the Creative Commons Attribution Non-Commercial License (http://creativecommons.org/licenses/by-nc/4.0) which permits unrestricted non-commercial use, distribution, and reproduction in any medium, provided the original work is properly cited.

(c) Copyright The Korean Institute of Electromagnetic Engineering and Science. All Rights Reserved. 
to inject calculated input phases were fabricated to verify the modified AF. The modified AF is described in Section II, and the simulated and measured far-field radiation pattern with a null are compared in Section III.

\section{MODIFIED AF OF CP CIRCULAR ARRAY}

Fig. 1(a) and (b) show the geometry of the CRPA circular array with and without consideration for the rotation angle of the $n^{t h}$ radiating element. A total radiation pattern can be obtained from an AEP multiplied by the AF. In this paper, the rotation angles of the radiating elements are considered in the AF instead of the AEP to expect the input phases for a radiation pattern's null before the design of radiating elements. The AF, having seven radiating elements without considering the rotation angle of the nth radiating element, can be expressed by Eq. (1):

$$
A F=\sum_{n=1}^{7} e^{j\left(k a \sin \theta \cos \left(\phi-\phi_{n}\right)+\beta_{n}\right)}
$$

Above, $a, \Phi_{n}$, and $\beta_{n}$ are at a distance between the center and the radiating element, an angle of the $n^{\text {th }}$ element from $\mathrm{x}$-axis, and an input phase of nth element, respectively. When the sev-

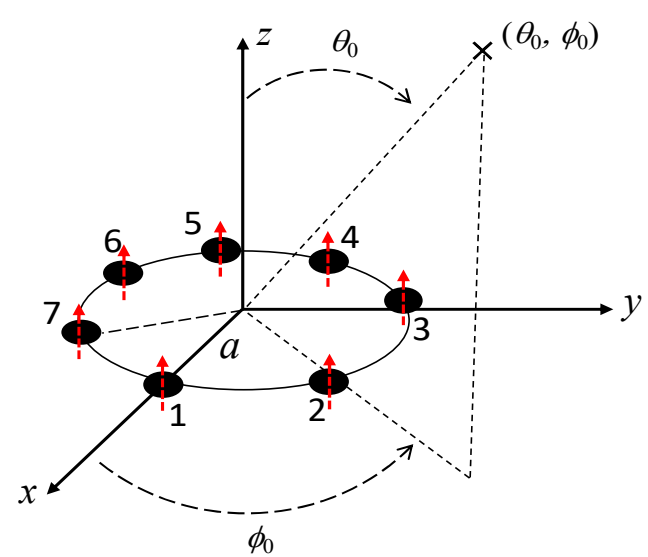

(a)

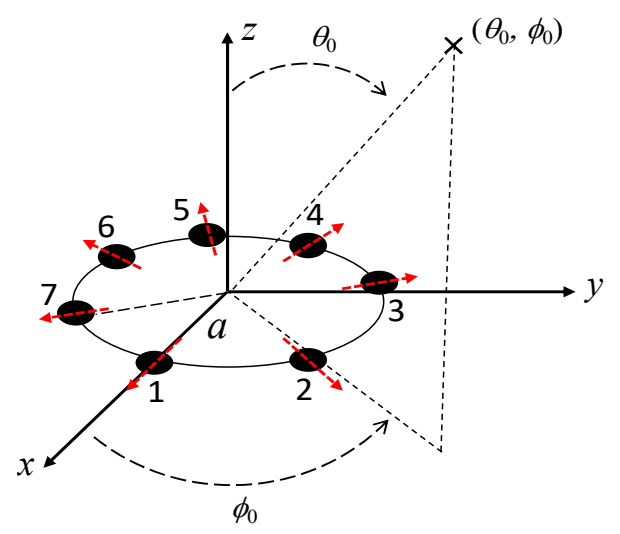

(b)

Fig. 1. Geometry of the CRPA circular array. (a) Without (b) with consideration of rotation angle of the $n^{t h}$ radiating element. en radiating elements are arranged toward the center and have $\mathrm{CP}$ characteristics, the AF of Eq. (1) can be modified as Eq. (2), because the rotation angle $\left(\psi_{n}\right)$ of the nth element can be expressed as the phase difference:

$$
A F=\sum_{n=1}^{7} e^{j\left(k a \sin \theta \cos \left(\phi-\phi_{n}\right)+\psi_{n}+\beta_{n}\right)}
$$

where $\psi_{\mathrm{n}}$ is $2(n-1) \pi / 7$ because the seven radiating elements are oriented toward the center. To achieve the pattern null at $\left(\theta_{0}\right.$, $\left.\Phi_{0}\right)$, Eq. (2) should be zero. Then, the input phase $\left(\beta_{n}\right)$ of the $n_{\text {th }}$ port can be derived as Eq. (3):

$$
\beta_{n}=-k a \sin \theta_{0} \cos \left(\phi_{0}-\phi_{n}\right)
$$

\section{SIMULATED AND MEASURED RESULTS}

The input phase $\left(\beta_{n}\right)$ of each port is calculated to achieve null steering in two directions (Case I: $\theta_{0}=0^{\circ}, \Phi_{0}=0^{\circ}$ and Case II: $\left.\theta_{0}=55^{\circ}, \Phi_{0}=150^{\circ}\right)$. Table 1 shows the calculated input phase $\left(\beta_{n}\right)$ from the modified AF. Fig. 2(a) and (b) show the CRPA array, which is introduced in [5] using seven MZR radiating elements. To obtain the $\mathrm{CP}$ characteristic, two orthogonal MZR antennas realized by a gap and a shorting via are employed and have dual feeding structures with a phase difference of $90^{\circ}$. Fig. 3(a) and (b) present feeding networks using a power divider for Case I and Case II, respectively. To approach the same power at each port, the dividers, including a $50-\Omega$ termination port with eight output ports, are designed despite the seven radiating elements. The substrate utilized for the power dividers has a thickness of $1.6 \mathrm{~mm}$ and the permittivity of 10.2. The power dividers are designed using a quarter-wave transformer composed of $50 \Omega$ and $70 \Omega$ lines, which have a width of $1.404 \mathrm{~mm}$ and $0.485 \mathrm{~mm}$. Fig. 4 shows simulated and measured radiation patterns with a nulling at Case I and Case II. The results were obtained using the ANSYS HFSS and the full anechoic chamber. As shown in Fig. 4(a) and (b), the measured results are in agreement with the simulated results, except for a difference of $5^{\circ}$ in the $\theta$ of Case II. The minor null points of the measured radiation patterns are generated by coaxial cables for the other ports.

Table 1. Calculated input phases from the modified AF

\begin{tabular}{cccc}
\hline$\theta_{0}=0^{\circ}, \Phi_{0}=0^{\circ}$ & $\beta_{n}\left(^{\circ}\right)$ & $\theta_{0}=55^{\circ}, \Phi_{0}=150^{\circ}$ & $\beta_{n}\left(^{\circ}\right)$ \\
\hline Port 1 & 0 & Port 1 & 5.76 \\
Port 2 & 0 & Port 2 & -26.26 \\
Port 3 & 0 & Port 3 & -38.51 \\
Port 4 & 0 & Port 4 & -21.75 \\
Port 5 & 0 & Port 5 & 11.38 \\
Port 6 & 0 & Port 6 & 35.95 \\
Port 7 & 0 & Port 7 & 33.44 \\
\hline
\end{tabular}




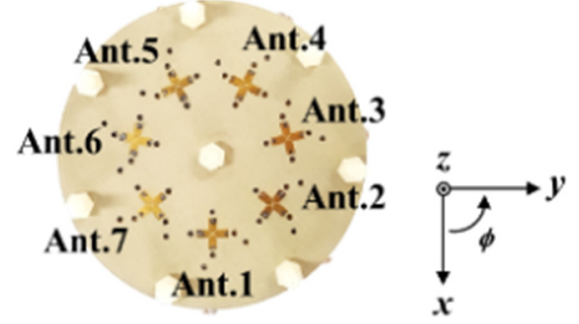

(a)
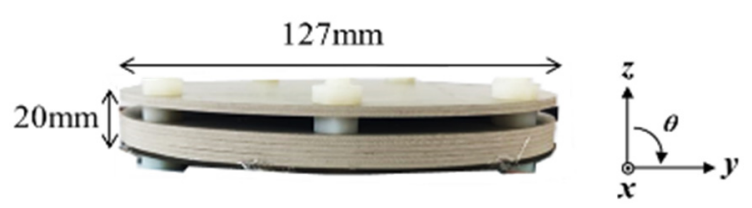

(b)

Fig. 2. Photographs of the fabricated CRPA array: (a) top view and (b) side view.

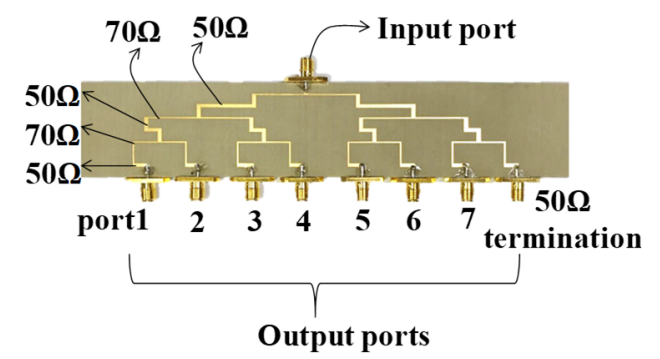

(a)

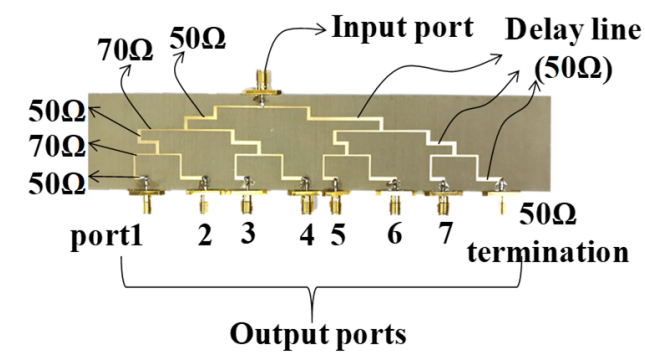

(b)

Fig. 3. Photographs of the fabricated feeding networks for nulling at (a) $\theta_{0}=0^{\circ}, \Phi_{0}=0^{\circ}$, (b) $\theta_{0}=55^{\circ}, \Phi_{0}=150^{\circ}$.

\section{CONCLUSION}

In this letter, a method to calculate the input phase of each port of radiating elements for a GPS anti-jam using a modified AF was presented. The modified AF considers both a rotation and an arrangement of radiating each element designed with circular array implemented by a MZR antenna with a CP characteristic. In addition, the power divider is employed to approach the calculated phases at each port. Null steering in a specific direction (Case I: $\theta_{0}=0^{\circ}, \Phi_{0}=0^{\circ}$ and Case II: $\theta_{0}=55^{\circ}, \Phi_{0}=150^{\circ}$ ) is confirmed by a simulated and measured radiation pattern with a null.

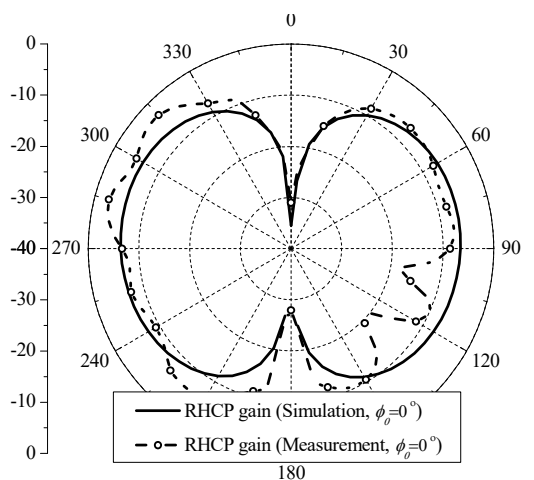

(a)

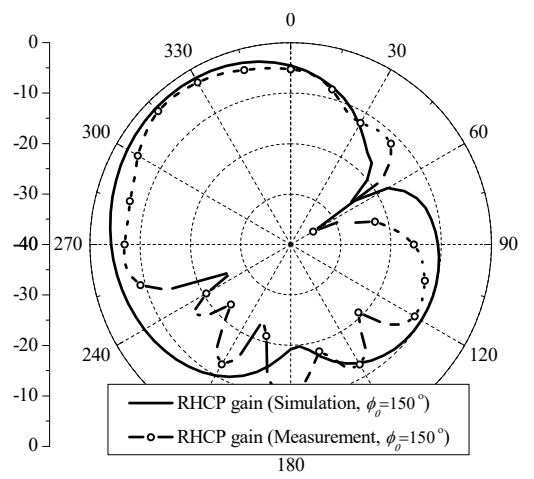

(b)

Fig. 4. Full-wave simulated and measured far-field radiation patterns ( $\Phi$ vs. dBi). (a) $\Phi=0^{\circ}$ plane, (b) $\Phi=150^{\circ}$ plane.

This research was supported by the Basic Science Research Program through the National Research Foundation of Korea (NRF) and funded by the Ministry of Education (No. 2015R1A6A1A03031833).

\section{REFERENCES}

[1] Y. D. Zhang and M. G. Amin, "Anti-jamming GPS receiver with reduced phase distortions," IEEE Signal Processing Letters, vol. 19, no. 10, pp. 635-638, 2011.

[2] J. R. Lambert, C. A. Balanis, and D. Decarlo, "Spherical cap adaptive antennas for GPS," IEEE Transactions on Antennas and Propagation, vol. 57, no. 2, pp. 406-413, 2009.

[3] R. L. Haupt and S. E. Haupt, "Phase-only adaptive nulling with a genetic algorithm," IEEE Transactions on Antennas and Propagation, vol. 45, no. 6, pp. 151-160, 1997.

[4] C. Zhang, X. Fu, L. P. Lighart, S. Peng, and M. Xie, "Synthesis of broadside linear aperiodic arrays with sidelobe suppression and null steering using whale optimization algorithm," IEEE Wireless Propagation Letters, vol. 17, no. 6, pp. 347-350, 2018.

[5] J. G. Lee, T. S. Kwon, B. H. Choi, and J. H. Lee, "Compact controlled reception pattern antenna (CRPA) array based on mu-zero resonance (MZR) antenna," IEICE Transactions on Communications, vol. 101, no. 6, pp. 1427-1433, 2018. 